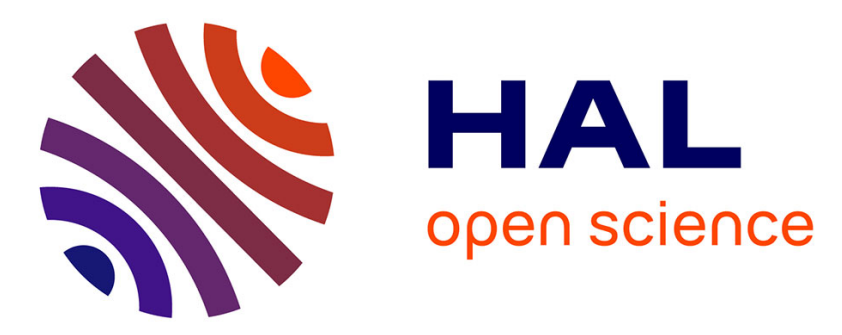

\title{
Conjectural Bifurcation Analysis of an Aircraft Engine Blade Undergoing 3D Unilateral Contact Constraints
}

\author{
Alain Batailly, Mathias Legrand
}

\section{To cite this version:}

Alain Batailly, Mathias Legrand. Conjectural Bifurcation Analysis of an Aircraft Engine Blade Undergoing 3D Unilateral Contact Constraints. ASME/IGTI Turbo Expo Conference, Jun 2014, Düsseldorf, Germany. 10.1115/GT2014-25674 . hal-01183448

\section{HAL Id: hal-01183448 \\ https://hal.science/hal-01183448}

Submitted on 10 Aug 2015

HAL is a multi-disciplinary open access archive for the deposit and dissemination of scientific research documents, whether they are published or not. The documents may come from teaching and research institutions in France or abroad, or from public or private research centers.
L'archive ouverte pluridisciplinaire HAL, est destinée au dépôt et à la diffusion de documents scientifiques de niveau recherche, publiés ou non, émanant des établissements d'enseignement et de recherche français ou étrangers, des laboratoires publics ou privés. 


\title{
Analyse de bifurcation conjecturale d'une aube de moteur d'avion sous contraintes unilatérales de contact en 3D
}

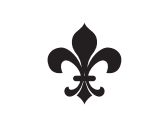

\author{
Alain Batailly \\ Laboratoire de Dynamique des Structures et Vibrations, Département de Génie Mécanique, \\ Université McGill, 817 rue Sherbrooke Ouest, Montréal, Québec H3A 0C3 \\ Mathias Legrand \\ Laboratoire de Dynamique des Structures et Vibrations, Département de Génie Mécanique, \\ Université McGill, 817 rue Sherbrooke Ouest, Montréal, Québec H3A 0C3
}

\section{Résumé}

La possibilité de prédire numériquement des interactions rotor/stator initiées par des contacts entre le sommet d'une aube et le revêtement abradable déposé sur le carter a été mise en évidence dans des études numériques récentes dont les résultats sont en accord avec des observations expérimentales. Notamment, la valeur des vitesses critiques, les zones endommagées de l'aube ainsi que le nombre de lobes d'usure dans le revêtement abradable ont pu être prédits avec précision pour un cas d'interaction entre une aube de compresseur basse-pression et un carter supposé parfaitement rigide. Le comportement dynamique de l'aube au voisinage de la vitesse d'interaction comporte toutefois encore de nombreuses zones d'ombre puisque des variations brutales des niveaux de vibration observés expérimentalement n'ont pas pu être prédits numériquement sans émettre des hypothèses contraires aux observations (telle qu'une perte de contact) ou irréalistes (une dégradation extrême des propriétés mécaniques du matériau abradable). Cet article se concentre sur l'étude du comportement vibratoire d'une aube de compresseur haute-pression à proximité d'une vitesse critique. Les scenarios d'interaction pour deux vitesses proches $\Omega_{c}$ et $\Omega_{c}^{*}$ sont analysés à l'aide d'une décomposition modale empirique des signaux numériques. Les résultats obtenus sont confrontés au scénario d'interaction tel que les profils d'usure calculés et les zones de touches prédites permettent de l'entrevoir. La nature instable de la réponse de l'aube lorsque la vitesse de rotation dépasse la vitesse critique est mise en avant et permet d'envisager un scénario plausible pour expliquer les brusques variations observées expérimentalement sans perte de contact.

e-mail: alain.batailly@mcgill.ca

website: http://structdynviblab.mcgill.ca/ 


\title{
Conjectural bifurcation analysis of an aircraft engine blade undergoing 3D unilateral contact constraints
}

\author{
Structural Dynamics and Vibration Laboratory, Department of Mechanical Engineering, McGill \\ University, 817 rue Sherbrooke Ouest, Montréal, Québec H3A 0C3
}

Alain Batailly

\section{Mathias Legrand}

Structural Dynamics and Vibration Laboratory, Department of Mechanical Engineering, McGill University, 817 rue Sherbrooke Ouest, Montréal, Québec H3A 0C3

\begin{abstract}
Prediction of rotor/stator interaction phenomena between a blade-tip and the surrounding abradable coating deposited on the casing has seen recent promising numerical developments that revealed consistency with several experimental set-up. In particular, the location of critical rotational frequencies, damaged blade areas as well as the wear pattern along the casing circumference were accurately predicted for an interaction scenario involving a low-pressure compressor blade and the surrounding abradable coating deposited on a perfectly rigid casing. The structural behaviour of the blade in the vicinity of a critical rotational frequency however remains unclear as brutal amplitude variations observed experimentally could not be numerically captured without assuming contact loss or an improbable drastic and sudden change of the abradable coating mechanical properties during the interaction. In this paper, attention is paid to the structural behaviour of a high-pressure compressor blade at the neighbourhood of a critical rotational frequency. The interaction scenarios for two close rotational frequencies: $\Omega_{c}$ and $\Omega_{c}^{*}$ are analyzed using empirical mode decomposition based on an adjusted B-spline interpolation of the time responses. The obtained results are compared to the interaction scenario dictated by the abradable coating removal history and the location of contact areas. The unstable nature of the blade vibratory response when the rotational frequency exceeds a critical rotational frequency is underlined and a plausible scenario arises for explaining a sudden and significant decrease of the blade amplitude of vibration without contact separation.
\end{abstract}

e-mail: alain.batailly@mcgill.ca

website: http://structdynviblab.mcgill.ca/ 


\section{Introduction}

The investigation of rotor/stator interactions stemming from structural contacts between blade tips and the surrounding casing — with or without abradable coating — has been the focus of several numerical and experimental studies related to the aerospace industry [1] as well as the power industry [2]. These works underline the complexity of such phenomena that may involve a single blade [3, 4], one full bladed disk assembly [5] or even several stages of the engine when precessional shaft motions occur and thus threaten the engine structural integrity. The growing interest in such phenomena is a consequence of the need for reduced operating clearances which maximize the engine efficiency but also lead to more frequent structural contacts. For each of these phenomena, the rotational frequency has been identified as a fundamental parameter. In particular, a rotational frequency is called critical if large amplitudes of vibrations occur with potential consequences over the turbomachine nominal operating conditions (blade failure, blade loss...).

In this context, the paper focuses on rotor/stator interactions between a single blade of a high-pressure compressor and the surrounding abradable coating deposited along the casing circumference. The analysis of this kind of interaction is relevant from an industrial standpoint since several manufacturers have witnessed such incidents [3,6]. More particularly, this paper brings further developments to the numerical work presented in [7] and related to experimental work carried out in [3]. Numerical simulations detailed in [7] highlight that it is now possible to accurately predict the location of a critical rotational frequency for which both the blade dynamics and the wear pattern on the abradable coating are properly captured. However, experimental observations made in [3] involve sudden variations of the blade vibratory response that cannot be explained numerically. A scenario based on a brutal and very large alteration of the abradable material properties is investigated in [7] where the blade vibratory response does feature sudden variations similar to those observed experimentally. However, this scenario remains unsatisfying due to the facts that : (1) it implies permanent contact loss contrary to experimental observations and (2) the scale of the abradable coating material properties alteration does not seem realistic.

Accordingly, further investigations are required in order to better apprehend the possible cause of these variations in the blade vibratory response. One of the key elements in the analysis provided in this paper concerns the post-processing strategy. Contrary to the approach used both experimentally [3] and numerically [7] — which is essentially based on a Fourier analysis of the time responses, assumed periodic after a sufficient number of blade revolutions- the analysis of time reponses is here expanded to transient time responses based on a modified Empirical Mode Decomposition (EMD) procedure. The EMD is an empirically based data-analysis method central to many recent numerical developments in signal analysis $[8,9]$. As such, it is particularly well suited for the analysis of non-stationary and nonlinear time responses eventhough its mathematical foundation partially remains an open question [10]. Signals are decomposed over a basis of Intrinsic Mode Functions

(IMF) featuring specific mathematical properties such as completeness and orthogonality. In the field of turbomachinery, EMD based methods such as the Local Mean Decomposition (LMD) [9] 
have been extensively applied for monitoring and diagnosis purposes [11] focusing essentially on their applicability to complicated time responses. The EMD is here used with an adjusted procedure for the computation of the IMF based on B-spline interpolation of local extrema of the time signal. This modification allows for an increased convergence rate on the analysed signals.

The contact scenario of interest in this paper is similar to the one in [7] and is briefly recalled in the first section along with the modeling of the abradable coating and the blade. The EMD postprocessing procedure is then introduced and details are given regarding the proposed modification of the method in order to increase its convergence rate. Results of the contact simulations are then presented globally in the third section of the paper. Two rotational frequencies are used for detailed investigations in the vicinity of a critical rotational frequency: $\Omega_{c}$ and $\Omega_{c}^{*}$ respectively a rotational frequency for which very high levels of vibration are observed and a rotational frequency very close to $\Omega_{c}$ but for which the amplitude of vibration is particularly low. Extensive time and frequency domain analyses of the results are provided in section five. Finally, the last section focuses on the analysis of the presented results and describes a plausible scenario for sudden drops of the amplitude of the blade vibratory response.

Note: For the sake of confidentiality, all numerical data have been normalized and units are not provided.

\section{Configuration of interest}

The blade of interest is located in the high-pressure compressor of an aircraft engine. The surrounding casing is assumed to be deformed along a two-nodal diameter free vibration mode-which means it is ovalized-and sufficiently rigid not to be sensitive to the blade impacts on the abradable coating. Consequently, the casing is modeled as a perfectly rigid mathematical profile on which is deposited the abradable coating. The imposed deformation of the casing was identified in [7] as one of the fundamental conditions in order to witness blade/abradable coating interaction.

\subsection{Structural considerations and modeling}

The blade is clamped on its root as pictured in Fig. 1. Its finite element model contains 13,803 nodes and 7,388 tetrahedron quadratic finite elements. In order to reduce computation times, a reduced order model of the blade accounting for centrifugal stiffening [7] is computed. Eight boundary nodes are considered for contact treatment, these nodes are evenly spaced along the blade from the leading edge to the trailing edge. Both modal convergence and convergence of the time integration algorithm are checked prior to any investigation but are not detailed here for the sake of brevity.

\subsection{Abradable coating}

The modeling of the abradable coating relies on the use of abradable plastic elements previously introduced in [12] and used in [7]. The initial clearance between the blade tip and the abradable 


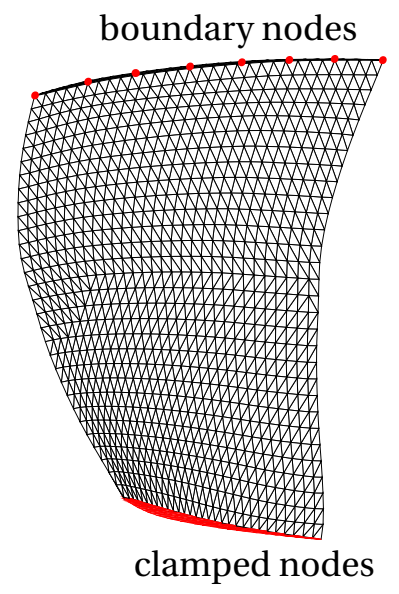

Figure 1: Finite element model of the blade

coating is constant (and equals $0.15 \%$ of the blade height) along the blade tip when the blade is at rest. The displacement of the blade tip due to centrifugal stiffening as well as the casing deformation are progressively applied over the first five revolutions of the blade in order to avoid initial blade/coating penetrations.

\section{Empirical mode decomposition}

The EMD consists of a decomposition of a time signal $y(t)$ - potentially nonlinear $^{1}$ and nonstationary-into a finite sum of Intrinsic Mode Functions (IMF):

$$
y(t)=\sum_{i=1}^{\mathrm{N}} \operatorname{IMF}_{i}(t)+r(t)
$$

where $r(t)$ is a residual function from which no IMF can be extracted, such as a monotonic function. The IMF must satisfy two criteria in order to be valid. As stated in [8]:

1. in the whole data set, the number of extrema and the number of zero crossings must either equal or differ at most by one;

2. at any point, the mean value of the envelope defined by the local maxima and the envelope defined by the local minima is zero.

Mathematically, the IMF must also form a complete and orthogonal adaptative basis. While completeness is ensured by the definition given in Eq. (1), orthogonality is not theoretically proven and must be checked based, for instance, on the criteria provided in [8].

Obtaining an IMF is typically a double iterative process as shown in Fig. 2. Given a time signal $y(t)$, the key steps for the computation of $\operatorname{IMF}_{i}(t)$ involve:

\footnotetext{
${ }^{1}$ A nonlinear signal refers to the response of a nonlinear system.
} 
1. the computation of a mean function $m_{k}(t)$ based on the detection of the local extrema of the signal $s_{k}(t)$ (with $s_{0}(t)=y(t)$ ), see [9] for details, this process is known as the sifting process;

2. the obtention of the envelope functions $a_{k}(t)$.

The computation of the envelope functions $a_{k}(t)$ may slightly differ depending on the context in which the EMD is performed. As an example, their acquisition is associated to a moving average algorithm in the LMD [9] while it is based on a B-spline interpolation of local extrema in the HilbertHuang transformation [8]. Same as for the Hilbert-Huang transform, it is here proposed to perform

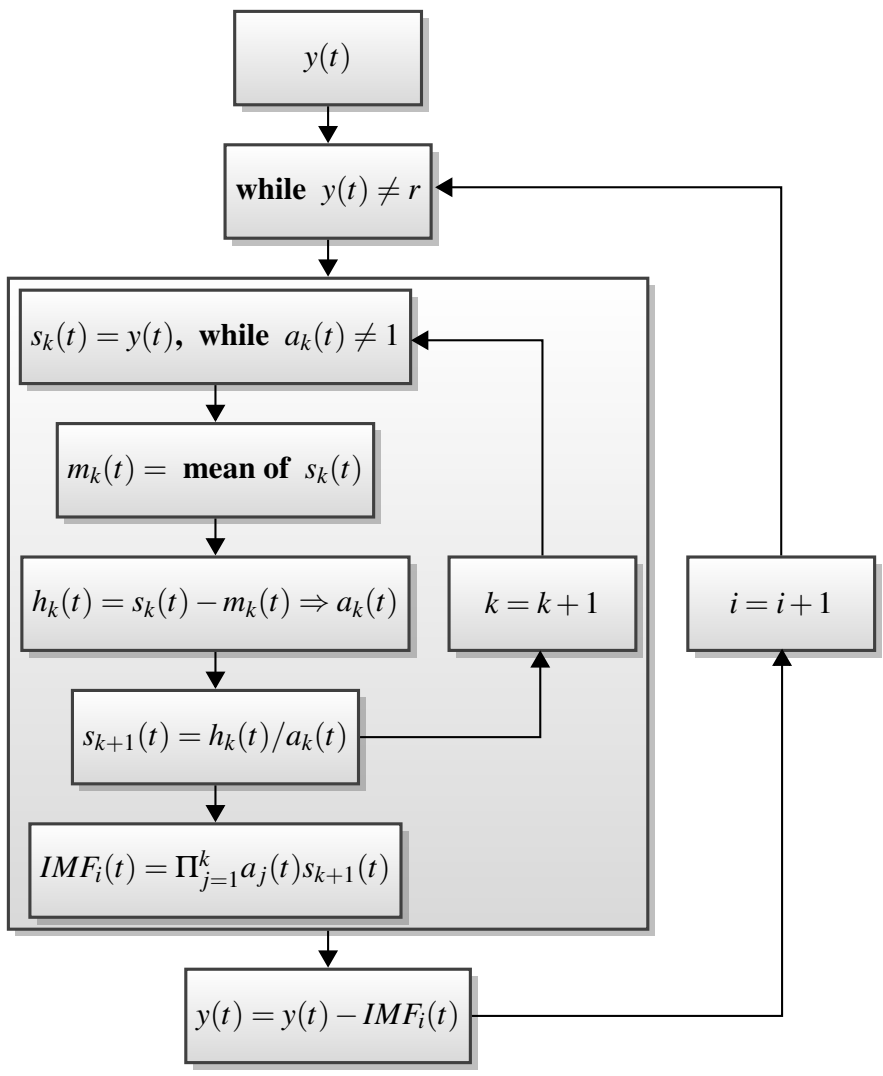

Figure 2: Principle of the emd

a B-spline interpolation of the local extrema of the time signal while ensuring the symmetry of the two envelopes with respect to the axis $y=0$ (the one of the local maxima and the one of the local minima) as featured by the LMD; this is achieved by considering local maxima as well as local minima - if they are negative-for the B-spline interpolation of the envelopes. In the following, the validity of the computed IMF with respect to the two criteria stated above as well as the orthogonality criterion stated in [8] is always verified but not detailed for the sake of brevity. 


\subsection{Validation}

The considered test signal is $y(t)=t^{2} \sin \left(t^{2}\right)$ over the interval $t \in[0 ; 16] \mathrm{s}$, and is plotted in Fig. 3(a). This signal features a linear growth of its frequency over time and a quadratic growth of its amplitude. The EMD is applied and only one non-negligible intrinsic mode function $\operatorname{IMF}_{1}(t)$ is obtained. The first two IMF functions are plot next to the associated instantaneous frequency-obtained with a Hilbert transform of the IMF- $\mathrm{IF}_{1}(t)$ and $\mathrm{IF}_{2}(t)$ in Figs. 3(b) and 3(c). It can be seen that $\mathrm{IMF}_{1}(t)$

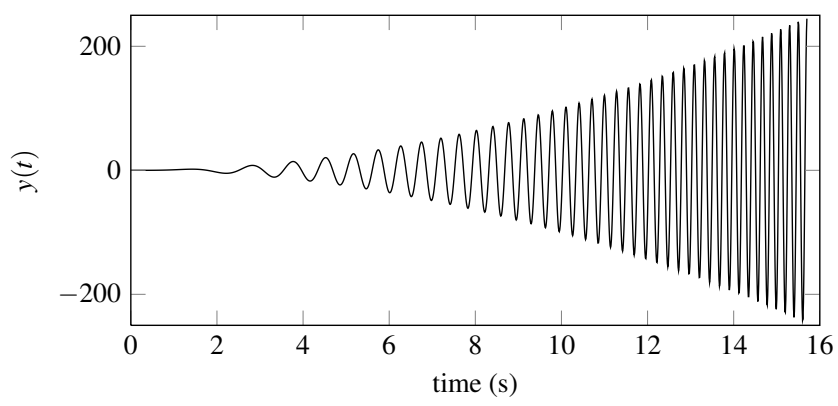

(a) Test signal

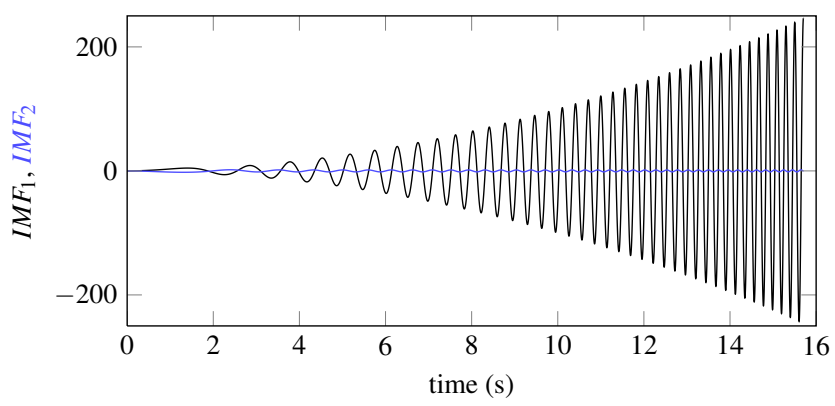

(b) Intrinsic mode functions $\mathrm{IMF}_{1}$ and $\mathrm{IMF}_{2}$

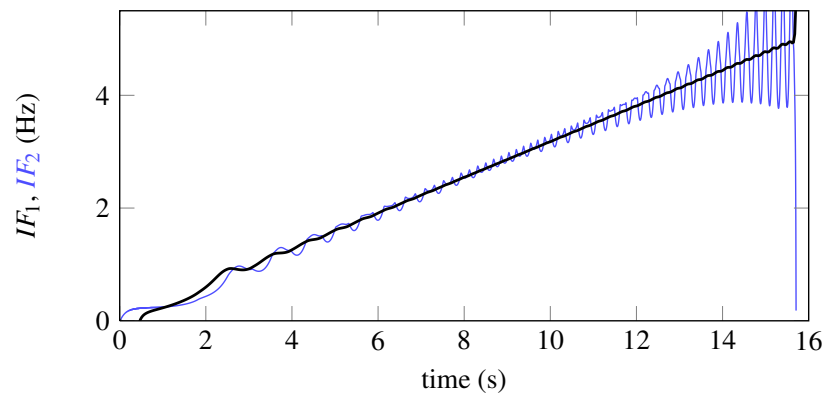

(c) Instantaneous frequencies $\mathrm{IF}_{1}$ and $\mathrm{IF}_{2}$

Figure 3: EMD of the test signal accurately represents the test signal and that the instantaneous frequency $\operatorname{IF}_{1}(t)$ is indeed linear in agreement with the test signal. Through this very simple example, a weakness of the EMD is underlined: its sensitivity to edge effects as illustrated in the oscillations of both $\operatorname{IMF}_{1}(t), \operatorname{IF}_{1}(t)$ and $\mathrm{IF}_{2}(t)$ for $t<2$ and $t>14$. Several numerical methods, such as waveform matching and mirroring techniques [10], exist to mitigate these effects. However, considering the very large amount of data 
points of each time response post-processed in the following, these effects are assumed to be very localized and thus negligible in this paper.

\section{Global results}

The employed numerical strategy relies on a central finite differences time integration scheme combined with a Lagrange multiplier approach for contact management. The $3 \mathrm{D}$ finite element model of the blade is reduced thus decreasing computation times, it accounts for centrifugal stiffening. Contact simulations are carried out over 750 blade revolutions for a properly selected

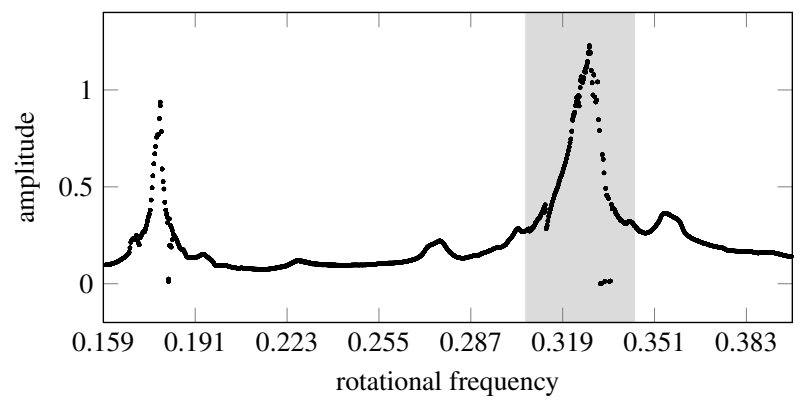

(a) Full rotational frequency range

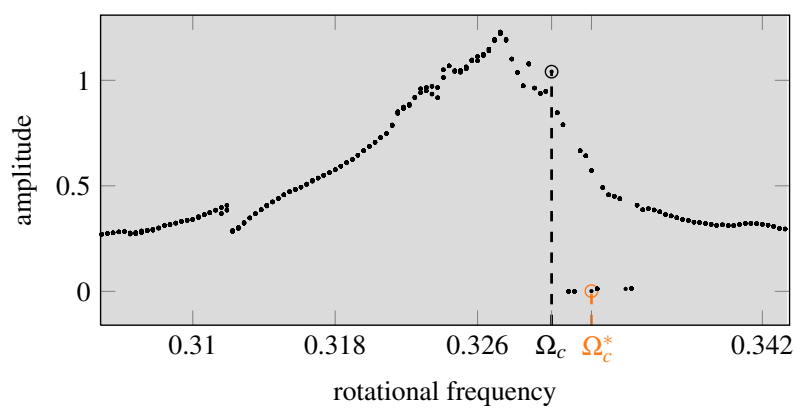

(b) Zoom over the second peak

Figure 4: Bifurcation diagrams

rotational frequency range. For each simulation a steady state is reached. This is confirmed by the bifurcation diagram plot in Fig. 4(a). Indeed, in this figure, the last 20 periods (of duration T) of the time responses are considered: the maximum displacement $u\left(\mathrm{~T}_{0}\right)$ computed over the first of these periods as well as all the displacements $u\left(\mathrm{~T}_{0}+i \mathrm{~T}\right), i=1, \ldots, 20$ appear as black marks. For each rotational frequency, the fact that almost only one mark is visible underlines the steadiness of the blade motion over time. Two peaks are visible in Fig. 4(a), the choice is made to focus on the peak obtained for $\Omega \in[0.307 ; 0.344]$. Corresponding results are more clearly shown in Fig. 4(b). The peak occurs for $\Omega \simeq 0.326$ which corresponds to a fourth of the first eigenfrequency of the blade. As mentioned in previous works, rotational frequencies $\Omega$ for which $\exists n, \Omega=f_{1} / n$ are typical 
locations ${ }^{2}$ of peaks of amplitudes [13]. It is also expected to witness $n$ privileged contact areas along the circumference of the casing. A more surprising outcome lies in the existence of steady-state solutions with very low-most of them strictly positive, meaning there is no contact loss-amplitude of vibration for rotational frequencies slightly above the interaction frequency.

The observation of very close stable solutions with very different levels of amplitude calls for thorough investigations and two rotational frequencies are considered in the following: $\Omega_{c}$ for which very high amplitude of vibrations are witnessed and $\Omega_{c}^{*}$ for which remarkably low levels of vibration occur.

\section{Witnessed interactions}

For both rotational frequencies $\Omega_{c}$ and $\Omega_{c}^{*}$, time responses are plotted and privileged contact areas along the casing circumference are shown. Both types of quantities highlight the fundamental differences between the two simulations. The full time responses and the steady state are shown in Fig. 5(a) and 5(b) for $\Omega_{c}$ and in Figs. 6(a) and 6(b) for $\Omega_{c}^{*}$. Steady states are detected based on a cross-correlation procedure in order to ensure that the signal is periodic, focusing on the evolution of its amplitude as well as its frequency content. The comparison of signals plotted in Figs. 5(a) and 6(a) reveals very distinct vibratory behaviours happening for two close rotational frequencies. For $\Omega_{c}$, the amplitude of vibration grows over time after contact is initiated until a steady state is reached. However, for $\Omega_{c}^{*}$ the amplitude of vibration grows after contact initiation, then stabilizes before undergoing a sudden drop around revolution 460. The contact areas maps in Figs. 5(c) and 6(c) provide additional elements for the analysis of these interactions. It is first visible that contact occurs almost simultaneously on the leading edge and the trailing edge with two contact areas per revolution between revolutions 0 and 200. With the increasing amplitude of vibration, comes a time for which the blade impacts the casing between the two main contact areas as visible after the $200^{\text {th }}$ revolution in Fig. 5(c). It is noticeable that these additional contact areas first appear on the trailing edge. Once the four contact areas are detected, the vibration level slowly stabilizes until steady state is reached for $\Omega_{c}$. A whole different scenario occurs for $\Omega_{c}^{*}$. As visible in Fig. 6(c), two additional contact areas sporadically appear around revolutions 175 and 340. However, these additional contact areas only appear at the trailing edge, the vibration level of the blade is too low to initiate contact at the leading edge. Because contact does not occur on the leading edge, the blade dynamics is not synchronous anymore with its rotational frequency and the interaction condition cannot be satisfied.

In order to better apprehend the blade dynamics during this key synchronization step (between revolutions 200 and 300 ), an extensive analysis of the time responses is carried out in the following.
Thionship is referred to as the interaction condition in the following. 


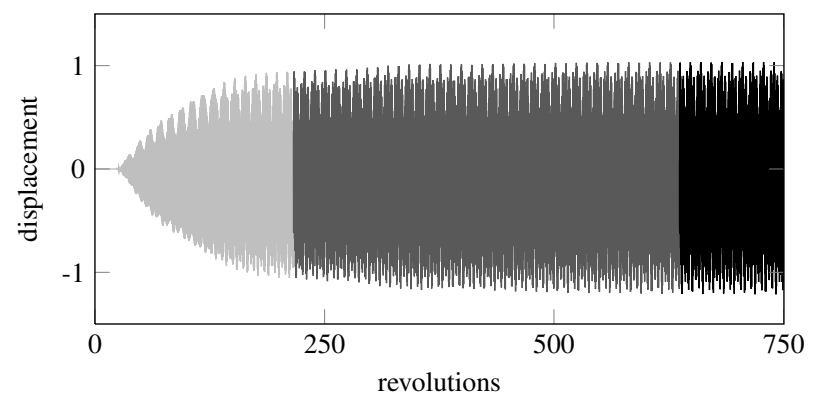

(a) Radial displacement of the leading edge: transient (-), steady frequency content $(-)$, steady state $(-)$

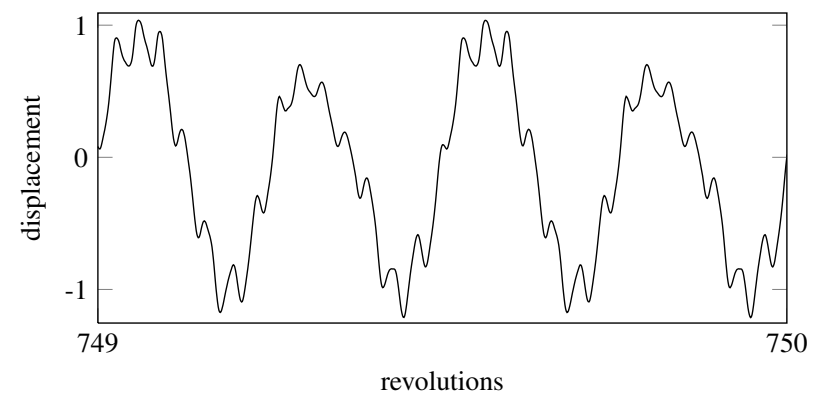

(b) Radial displacement of the leading edge once steady state is reached
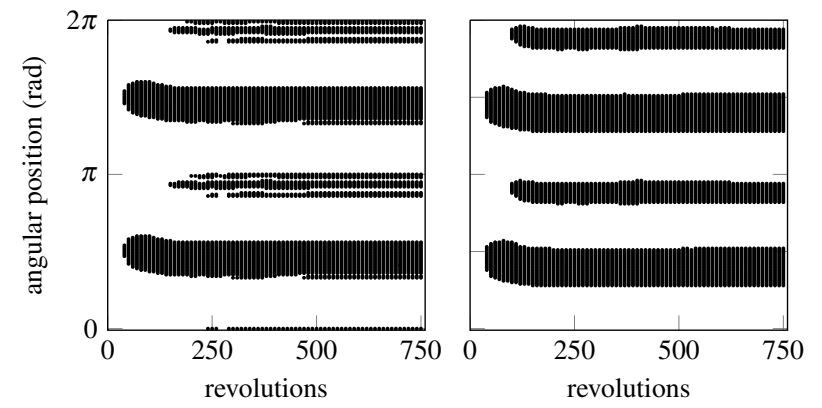

(c) Contact locations, left: leading edge, right: trailing edge

Figure 5: Predictions at $\Omega_{c}$

\section{Blade response analysis}

\subsection{Fourier transform}

Since steady states exist both rotational frequencies, a Fourier transform of the responses may be performed. The spectrum obtained for $\Omega_{c}$ and $\Omega_{c}^{*}$ are respectively pictured in Figs. 7(a) and 7(b). Based on the rotational frequency of the blade and the number of predicted contact areas, it is possible to determine for each interaction the fundamental excitation frequency: $f_{c}=\mathrm{N}_{l} \Omega$ where $\mathrm{N}_{l}$ is the number of lobes. Because the casing is ovalized, $\mathrm{N}_{l}=2$ in this paper. The location of the peaks obtained in each spectrum is perfectly consistent with the fundamental excitation frequency 


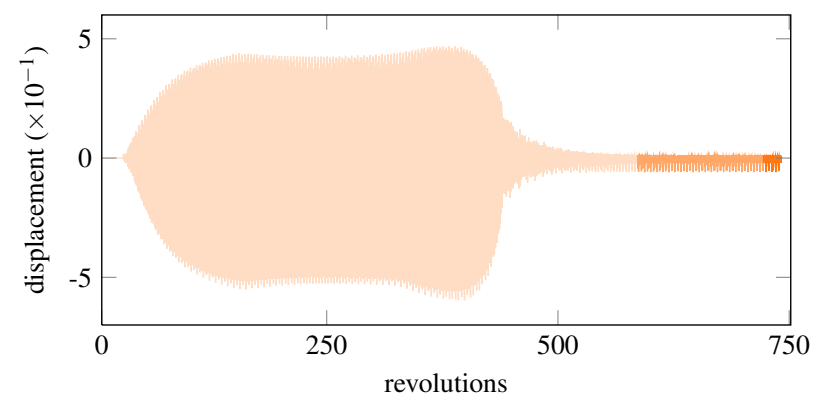

(a) Radial displacement of the leading edge: transient ( - ), steady frequency content $(-)$, steady state $(-)$

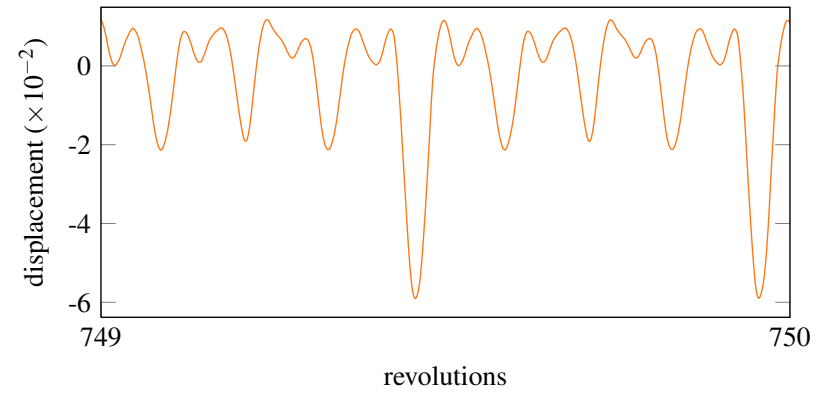

(b) Radial displacement of the leading edge once steady state is reached
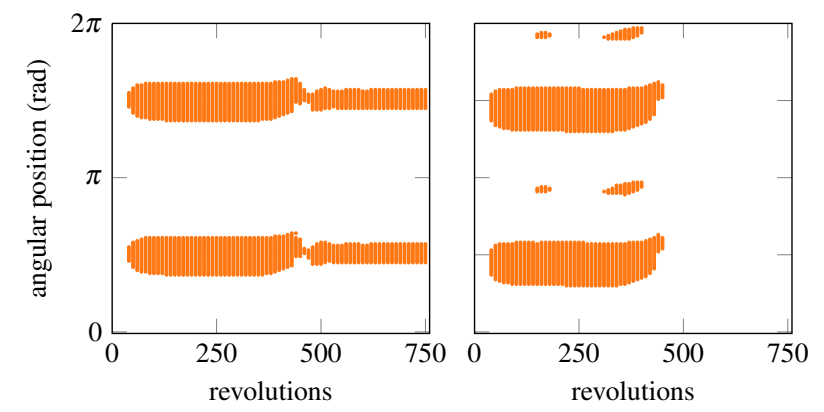

(c) Contact locations: left: leading edge, right: trailing edge

Figure 6: Predictions at $\Omega_{c}^{*}$

and its superharmonics as shown in Figs. 7(a) and 7(b). Peaks amplitudes highly differ from a spectrum to another-one may note the different order of magnitude used on the vertical axis between these two figures. However, these spectrum fail to provide an explanation regarding the origin of these differences. For that reason, the EMD of the full time responses is carried out with a particular emphasis between revolutions 200 and 300 where time responses are still transient. Other strategies involving wavelets or spectrograms (with short time Fourier transform) could also be considered. However, wavelets may lead to results arduous to interprete and spectrograms are highly sensitive to the length of the time window. 


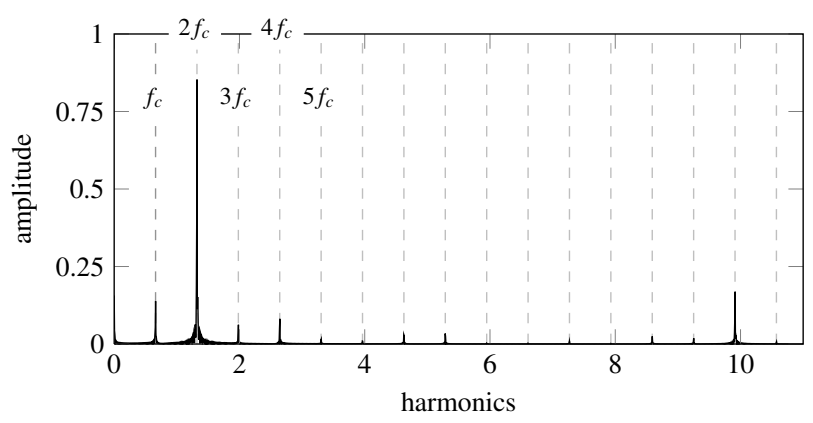

(a) response at $\Omega_{c}$

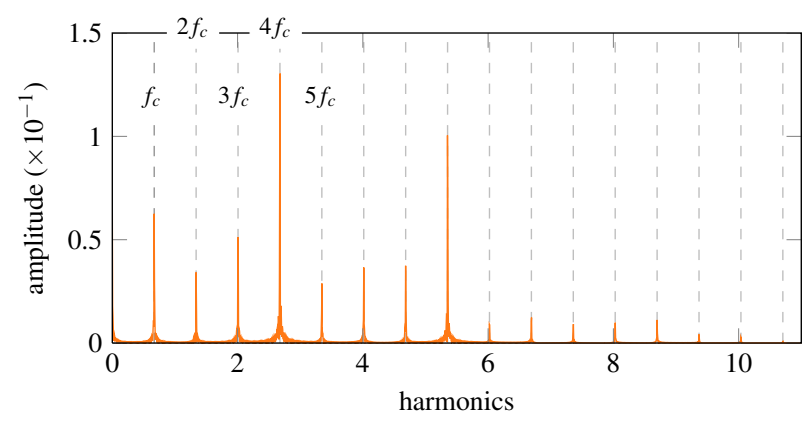

(b) response at $\Omega_{c}^{*}$

Figure 7: Spectra of the steady states

\subsection{Application of EMD}

The EMD of the time responses plotted in Figs. 5(a) and 6(a) is carried out and the first six IMF are given for each signal in Figs. 8 and. 9. Higher order IMF feature negligible amplitudes and are not considered in this study. Interaction at $\Omega_{c}$ is almost entirely represented by its second IMF while at $\Omega_{c}^{*}$, the dominant IMF is $\mathrm{IMF}_{1}$, at least for the 300 first revolutions. By construction, the firsts IMF of a signal are essentially representative of the signal high frequency content. As the empirical mode decomposition is processed, IMF progressively reveal lower frequency content. In the light of this remark, the two interactions differ significantly in terms of their high frequency content.

For the rotational frequency $\Omega_{c}$, the amplitude of the computed IMF are remarkably stable through out the simulation: only minor variations are visible during the first 100 revolutions due to contact initiation and small amplitudes are detected once steady state has been reached for $\mathrm{IMF}_{3}$ to $\mathrm{IMF}_{6}$. The time response almost entirely stems from three IMF which underlines the steadiness of the simulated interaction and corroborates the fact that the signal frequency content is steady after a few revolutions as shown in Fig. 5(a).

Similar observations can be made for rotation frequency $\Omega_{c}^{*}$ over the 300 firsts revolutions. After this threshold, the amplitude of $\mathrm{IMF}_{2}$ drops and the significant components are detected on $\mathrm{IMF}_{2}$ to $\mathrm{IMF}_{6}$. Between revolutions 200 and 300, which is the time interval that was identified previously as relevant for the blade synchronization, the instantaneous frequencies associated with the dominant 

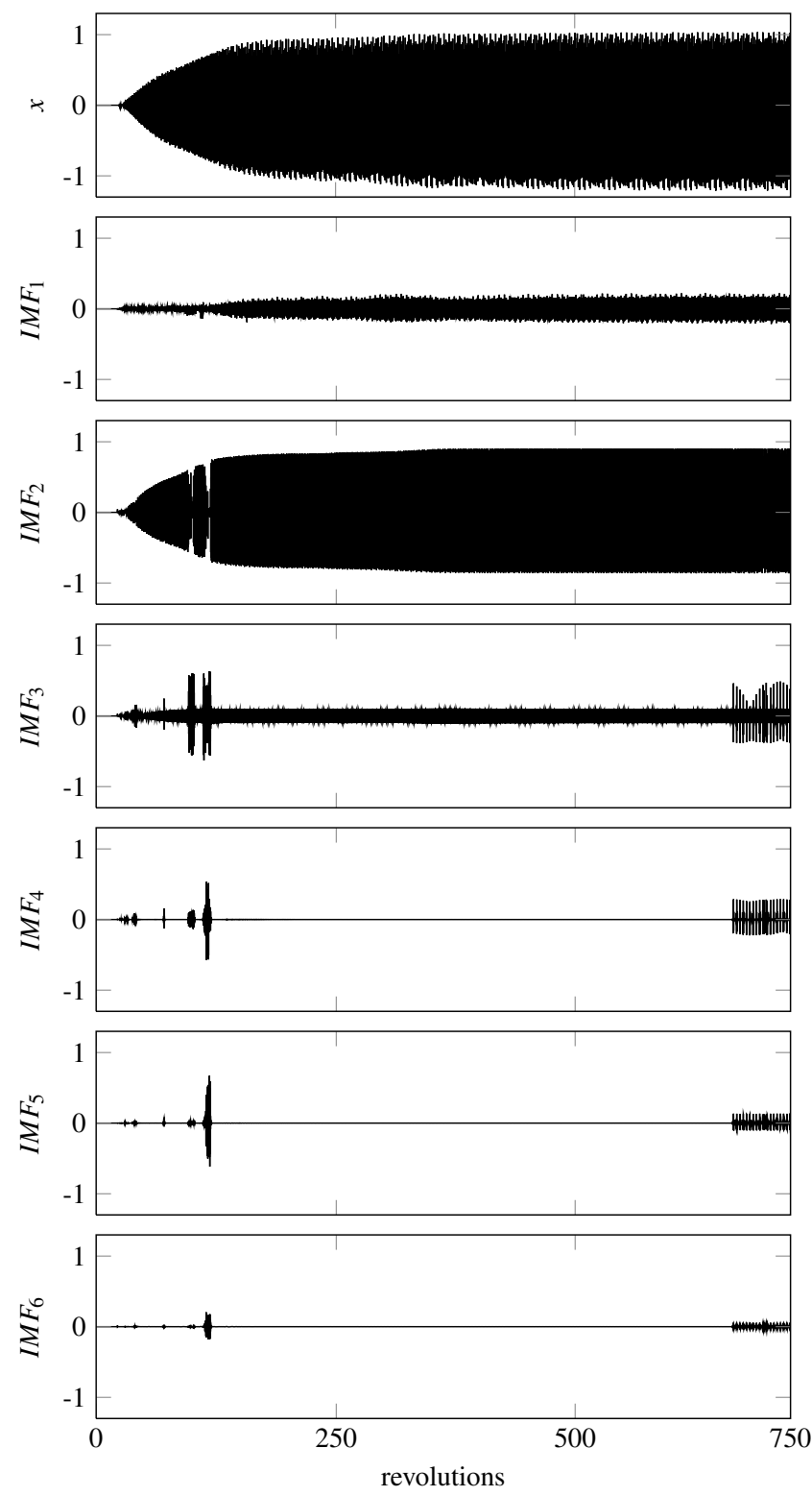

Figure 8: Time response and $\operatorname{six} \operatorname{imf}$ for $\Omega_{c}$

IMF of each signal are depicted in Figs. 10(a) and 10(b). Both signals are centered around the frequency $f=2 f_{c}$ in good agreement with the contact configuration and confirms the similarity of the two observed interactions.

\subsection{Hilbert spectrum}

In order to account for the influence of each IMF, the Hilbert spectrum of the time response obtained for $\Omega_{c}$ and $\Omega_{c}^{*}$ are respectively depicted in Fig. 11(a) and in Fig. 11(b). These spectra picture the evolution of the amplitude of each IMF in the frequency/time plane. Results are normalized with respect to $x^{*}$, defined as $x^{*}=\max (\|x(t)\|)$. The Hilbert spectrum obtained for $\Omega_{c}$ features two 

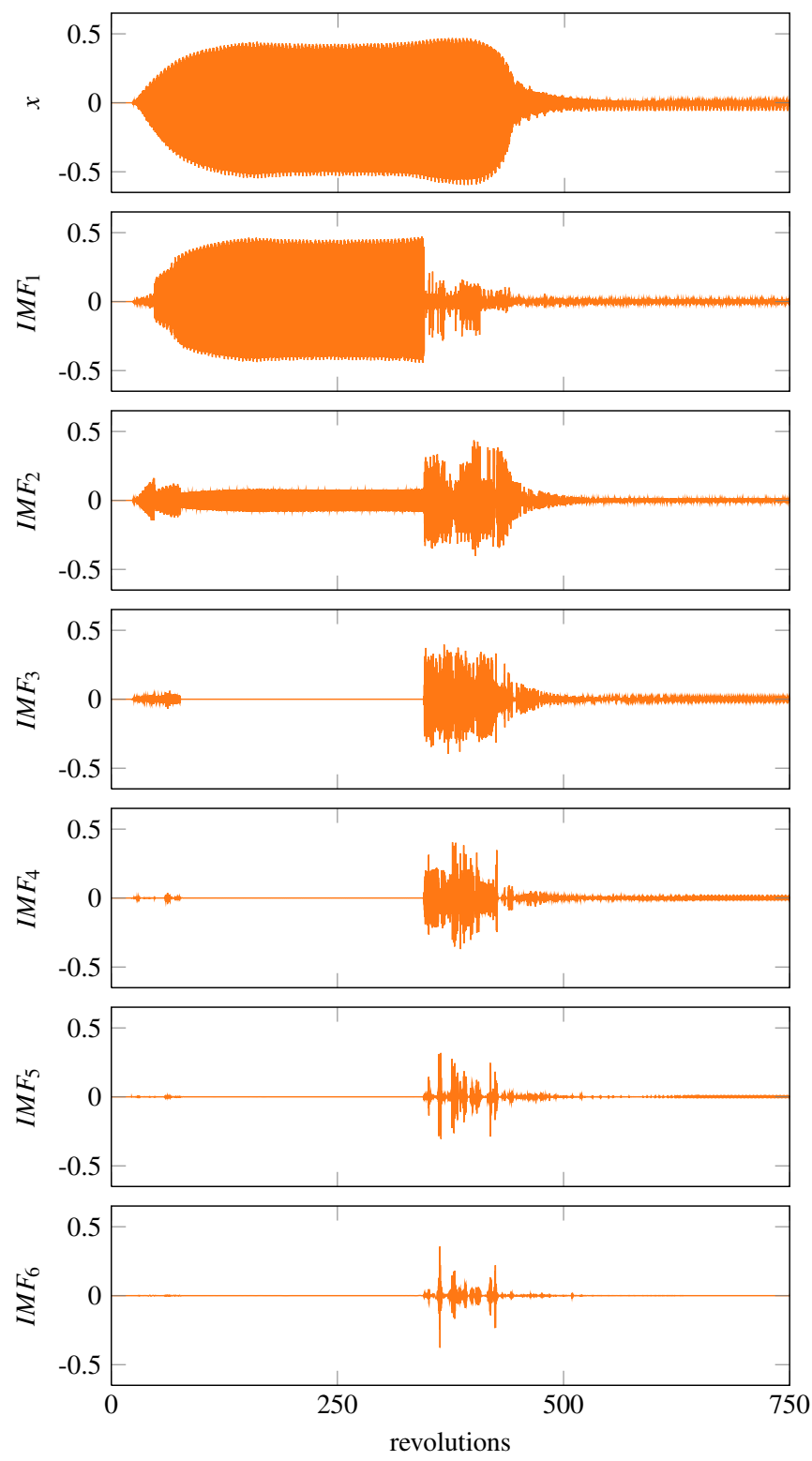

Figure 9: Time response and $\operatorname{six} \operatorname{imf}$ for $\Omega_{c}^{*}$

main frequency components: $f_{c}$ and $2 f_{c}$ as well as a third high frequency component $(\simeq 10$, also visible in the Fourier spectrum depicted in Fig. 7(a)) associated with $\mathrm{IMF}_{1}$. The latter is remarkably absent in the Hilbert spectrum for $\Omega^{*}$ in Fig. 11(b). In this spectrum, one can see two main frequency components $f_{c}$ and $2 f_{c}$ over the 300 first revolutions. The amplitude drop of $\mathrm{IMF}_{2}$ is visible here between revolutions 300 and 500 through a cloudy area with no dominant frequency. After 500 revolutions, the spectrum reveals five frequency components: $f_{c}, 2 f_{c}, 3 f_{c}, 4 f_{c}$ and $8 f_{c}$ in good agreement with the Fourier spectrum depicted in Fig. 7(b). 


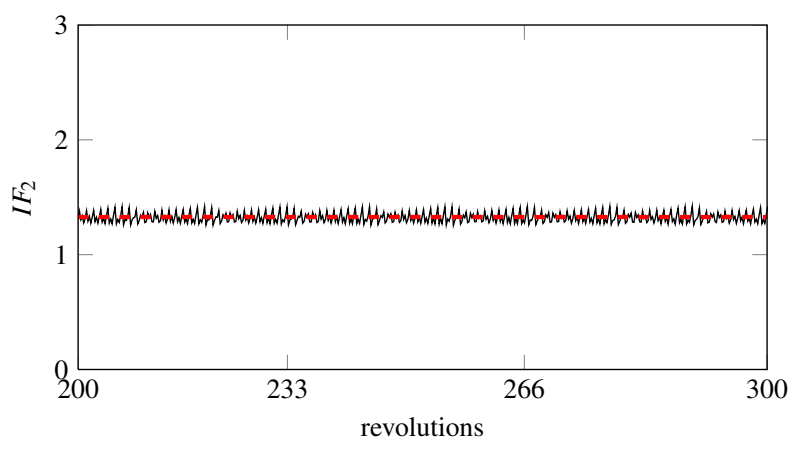

(a) $\Omega_{c}$

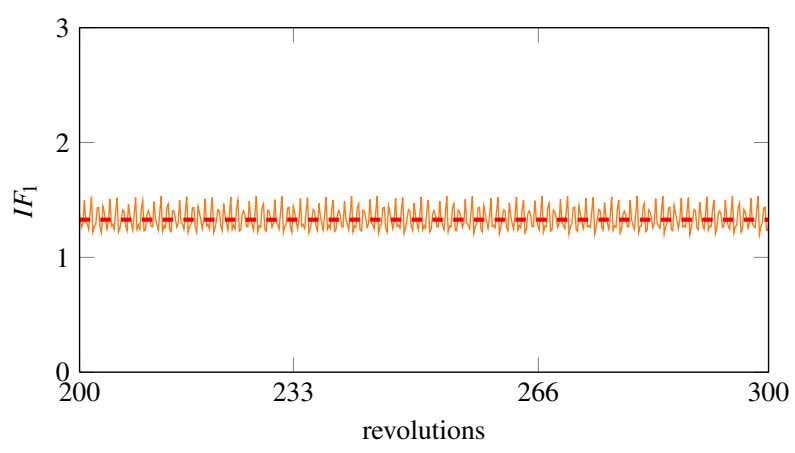

(b) $\Omega_{c}^{*}$

Figure 10: Instantaneous frequencies between revolutions 200 and 300, excitation frequency $2 f_{c}(---)$

\subsection{Conjectural bifurcation analysis}

Results obtained using the EMD of the time responses confirm that the fundamental difference between the interaction scenario at $\Omega_{c}$ and $\Omega_{c}^{*}$ lies in the synchronization of the blade vibration with respect to the casing deformation. However, the possibility of such synchronization does not solely depend on the rotational frequency since, as pictured in Fig. 4 (b) over $\Omega \in\left[\Omega_{c} ; \Omega_{c}^{*}\right]$, synchronized and non-synchronized steady states-leading to very high, very low amplitudes of vibration or even contact loss-alternate for very close rotational frequencies. These observations point out the fact that rotor/stator interaction scenarios may also critically depend on other physical parameters than the rotational frequency. In that sense, the bifurcation diagram plot in Fig. 4(b) could be enriched considering a third dimension relative to such parameter.

Based on existing numerical and experimental work, two parameters have been identified as playing a critical role in the blade vibration synchronization:

1. the angular position of contact initiation;

2. the initial ${ }^{3}$ clearance configuration (ICC) along the blade tip [14].

\footnotetext{
${ }^{3}$ Initial refers to the clearance configuration before contact and subsequent abradable removal occur.
} 


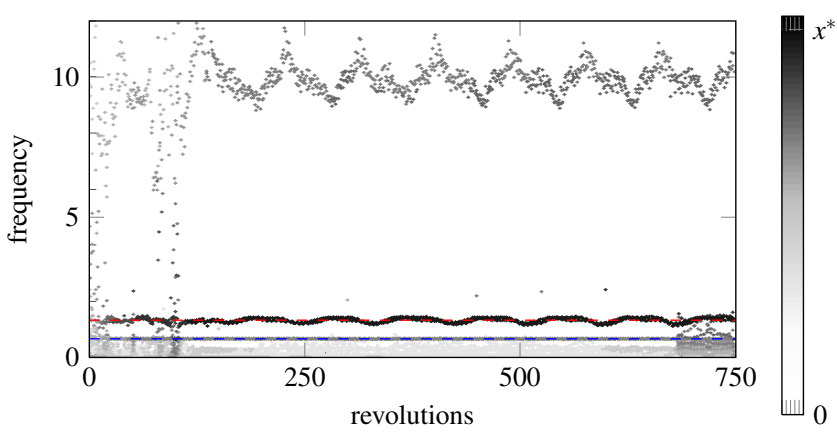

(a) $\Omega_{c}$

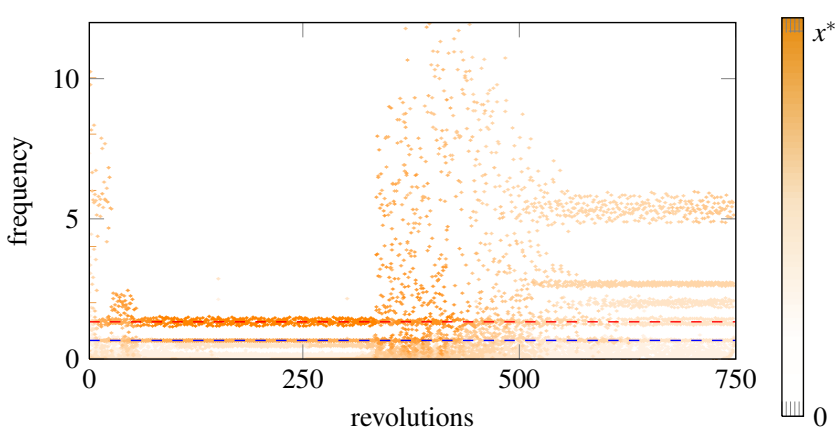

(b) $\Omega_{c}^{*}$

Figure 11: Hilbert spectra, fundamental excitation frequencies $f_{c}(---), 2 f_{c}(---)$

These two parameters are related to the rotational frequency in our study through centrifugal stiffening. For each rotational frequency, the blade stiffness is modified and so is the ICC. The modification of the ICC also impacts the location of the first blade/casing contact. Consequently, it is particularly arduous to assess the influence of these parameters independently from the rotational frequency.

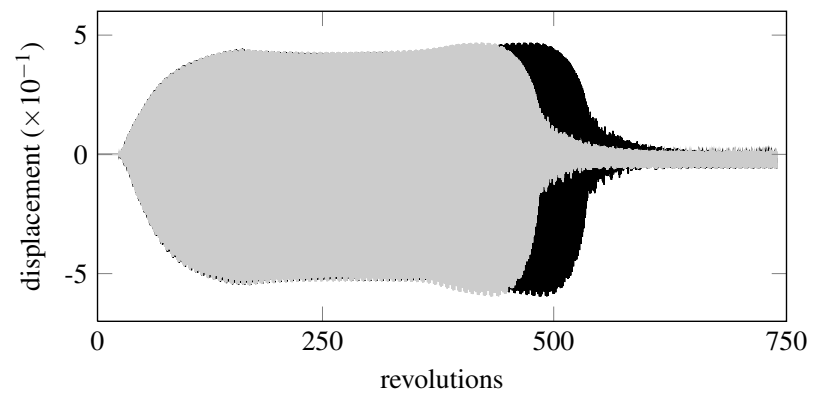

Figure 12: Time responses at $\Omega_{c}^{*}$ with different initial contact locations (angular shift $\Delta \theta=\pi / 4$ )

For a given rotational frequency, the influence of the angular position of the first blade/casing contact is found to have no influence over the amplitude of the obtained steady state. No matter the scenario, high amplitude of vibration such as $\Omega_{c}$, low amplitude of vibrations such as $\Omega_{c}^{*}$ or contact loss, the amplitude of the steady state seems independent of the angular position of the first contact. 
Though it is observed that this parameter has a non-negligible impact on the number of revolutions required to reach steady state. As an example, the drop in amplitude in Fig. 6(a) around the $400^{\text {th }}$ revolution is significantly shifted depending on where the first contact occurs as shown in Fig. 12.

Attention is now paid to the ICC. In previous simulations, the ICC is constant from the leading edge to the trailing edge. This configuration is used as a reference in this study and is shown in $(-)$ in Fig. 13(a). Based on the fact that a major difference between the contact scenarios at $\Omega_{c}$ and $\Omega_{c}^{*}$ lies in the number of contact areas on the leading edge (see Figs. 5(c) and 6(c)) it is proposed to modify the ICC at the leading edge of a value $\delta c$. The clearance modification at the middle of blade tip is $\delta c / 2$ and the clearance at the trailing edge is invariant. A quadratic interpolation is then used to compute the clearance for all the other contact nodes. As pictured in Fig. 13(a), seven clearance configurations, from $\delta c=-25 \%$ to $\delta c=+25 \%$ are considered.

For each ICC, contact simulations are limited to the rotational frequency range $\Omega \in\left[\Omega_{c} ; \Omega_{c}^{*}\right]$. Corresponding bifurcation diagrams are superimposed in Fig. 13(b) with the same colour code ${ }^{4}$. For a given rotational frequency, the critical influence of the ICC is patent since a modification of $5 \%$ of the clearance on the leading edge strongly affects the amplitude of the obtained steady state. The figure reveals two distinct branches in the bifurcation diagram for post-critical rotational frequencies: one featuring a mix of very low amplitudes of vibration (LAV) and contact loss motions and one with high amplitudes of vibration (HAV) that may threaten the blade structural integrity.

It seems reasonable to assume that for all types of blade (from the low-pressure compressor to the turbine) such dependence to the ICC exist. Because the progressive wear of the abradable coating dynamically drives the ICC, the drops observed in [3] could be a consequence of a minor clearance evolution that triggers a bifurcation of the blade response from the HAV branch to the LAV branch.

The results presented in this section call for more detailed investigations of the dependence of rotor/stator interactions to the ICC. So far, it seems that this dependence is exacerbated for rotational frequencies slightly higher than identified critical rotational frequencies. Close to the critical rotational frequency, meaning slightly below $\Omega_{c}$ in the context of this paper, this dependence is less clear since separated branches are not distinguishable.

\section{Conclusion}

Based on an existing numerical strategy for the simulation of rotor/stator interactions with abradable coating removal, this study focuses on the vibratory behaviour of a high-pressure compressor blade for rotational frequencies slightly above a critical rotational frequency. Global results are first presented over a properly selected rotational frequency range as a bifurcation diagram featuring very distinct amplitude of vibrations for very close frequencies thus hinting for possible bifurcations. An in-depth analysis of the obtained time responses is carried out with both Fourier transform and

\footnotetext{
${ }^{4}$ All simulations lead to a clear steady state thus for each rotational frequency, only one mark is visible per colour code in Fig. 13(b)
} 


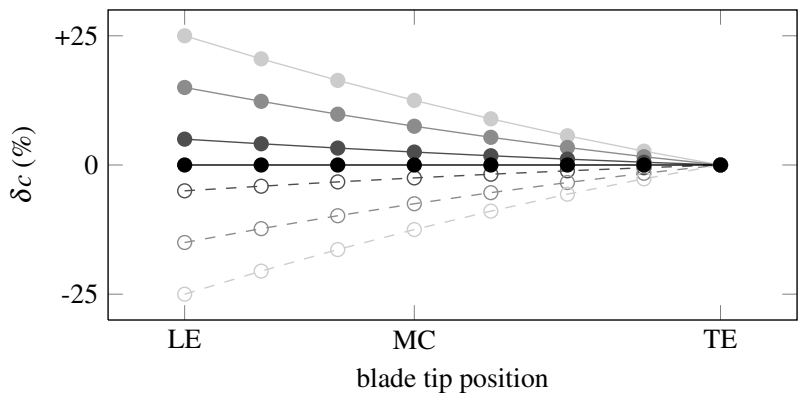

(a) ICC

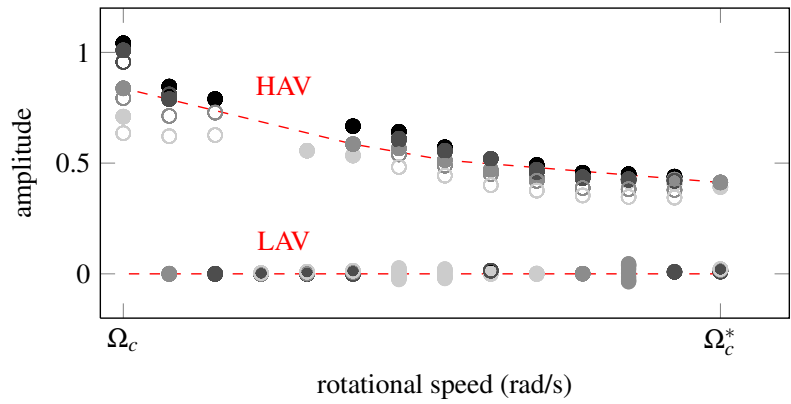

(b) BIFURCATION DIAGRAM (to be read by colour)

Figure 13: Influence of the icc

empirical mode decomposition. The assets of the latter are underlined and it is established that low amplitude of vibrations are the consequence of a non-synchronization of the blade vibration with the deformation of the casing. In particular, the establishment of contact at the leading edge seems key in the rise of interactions. Further investigations accounting for an evolution of the angular position of the initial blade/casing contact as well as the initial clearance configuration highlight the critical role of initial clearance in the blade vibratory response in the vicinity of a critical rotational frequency. Indeed, for a given rotational frequency, a minor modification of the clearance may impact the system going from a high amplitude of vibration steady state to contact separation.

\section{Acknowledgement}

Thanks go to Snecma for its technical and financial support. This work takes place in the framework of the MAIA mechanical research and technology program sponsored by CNRS, ONERA and SAFRAN Group.

\section{REFERENCES}

[1] S.K. Sinha. Rotordynamic analysis of asymmetric turbofan rotor due to fan blade-loss event with contact-impact rub loads. Journal of Sound and Vibration, 332:2253-2283, 2013. 
DOI:10.1016/j.jsv.2012.11.033.

[2] M. Torkhani, L. May, and P. Voinis. Light, medium and heavy partial rubs during speed transients of rotating machines: numerical simulation and experimental observation. Mechanical Systems and Signal Processing, 29:45-66, 2012. DOI:10.1016/j.ymssp.2012.01.019.

[3] A. Millecamps, J.F. Brunel, P. Dufrénoy, F. Garcin, and M. Nucci. Influence of thermal effects during blade-casing contact experiments. In Proceedings of the ASME IDETC/CIE-DETC200986842, San Diego, USA, September 2009. DOI:10.1115/DETC2009-86842.

[4] C. Padova, J. Barton, M. Dunn, and S. Manwaring. Experimental results from controlled blade tip/shroud rubs at engine speed. Journal of Turbomachinery, 129(4):713-723, 2007. DOI:10.1115/1.2720869.

[5] P. Schmiechen. Travelling wave speed coincidence. PhD thesis, College of Science, Technology and Medicine, London, UK, 1997.

[6] R.J. Williams. Simulation of blade casing interaction phenomena in gas turbines resulting from heavy tip rubs using an implicit time marching method. In Proceedings of the ASME Turbo Expo 2011-GT2011-45495, Vancouver, Canada, 2011. DOI:10.1115/GT2011-45495.

[7] A. Batailly, M. Legrand, A. Millecamps, and Garcin F. Numerical-experimental comparison in the simulation of rotor/stator interaction through blade-tip/abradable coating contact. Journal of Engineering for Gas Turbines and Power, 134, 2012. DOI:10.1115/1.4006446.

[8] N.E. Huang, Z. Shen, S.R. Long, M.C. Wu, H.H. Shih, Q. Zheng, N.-C. Yen, C.C. Tung, and H.H. Liu. The empirical mode decomposition and the hilbert spectrum for nonlinear and nonstationary time series analysis. Proc. R. Soc., 454:903-995, 1998. DOI:10.1098/rspa.1998.0193.

[9] J.S. Smith. The local mean decomposition and its application to eeg perception data. Journal of the Royal Society Interface, 2:443-454, 2005. DOI:10.1098/rsif.2005.0058.

[10] G. Rilling, P. Flandrin, and P. Gonçalvès. On empirical mode decomposition and its algorithms. In Proceedings of the $6^{\text {th }}$ IEEE/EURASIP Workshop on Nonlinear Signal and Image Processing, Grado, Italy, 2003.

[11] Y. Yang, J. Cheng, and K. Zhang. An ensemble local means decomposition method and its application to local rub-impact fault diagnosis of the rotor-systems. Measurement, 45:561-570, 2012. DOI:10.1016/j.measurement.2011.10.010.

[12] M. Legrand, A. Batailly, and C. Pierre. Numerical investigation of abradable coating removal through plastic constitutive law in aircraft engine. Journal of Computational and Nonlinear Dynamics, 7, 2011. DOI:10.1115/1.4004951. 
[13] M. Legrand, A. Batailly, B. Magnain, P. Cartraud, and C. Pierre. Full three-dimensional investigation of structural contact interactions in turbomachines. Journal of Sound and Vibration, 331(11):2578-2601, 2012. DOI:10.1016/j.jsv.2012.01.017.

[14] N. Salvat, A. Batailly, and M. Legrand. Modeling of abradable coating removal in aircraft engines through delay differential equations. Journal of Engineering for Gas Turbines and Power, in press:-, 2013. DOI:10.1115/1.4024959. 